University of New Hampshire

University of New Hampshire Scholars' Repository

Space Science Center

Institute for the Study of Earth, Oceans, and

Space (EOS)

1993

\title{
COMPTEL observations of the inner galaxy
}

\author{
H Bloemen \\ Space Research Organization of the Netherlands \\ H deBoer \\ SRON \\ R Van Dijk \\ University of Amsterdam \\ J W. den Herder \\ SRON \\ W Hermsen \\ Space Research Organization of the Netherlands (SRON)
}

See next page for additional authors

Follow this and additional works at: https://scholars.unh.edu/ssc

Part of the Astrophysics and Astronomy Commons

\section{Recommended Citation}

COMPTEL observations of the inner galaxy Bloemen, $\mathrm{H}$. and de Boer, H. and van Dijk, R. and den Herder, J. W. and Hermsen, W. and Kuiper, L. and Swanenburg, B. N. and de Vries, C. P. and Collmar, W. and Diehl, R. and Lichti, G. G. and Schönfelder, V. and Steinle, H. and Strong, A. W. and McConnell, M. and Connors, A. and Morris, D. and Stacy, G. and Bennett, K., AIP Conference Proceedings, 280, 30-34 (1993),

DOI:http://dx.doi.org/10.1063/1.44137

This Conference Proceeding is brought to you for free and open access by the Institute for the Study of Earth, Oceans, and Space (EOS) at University of New Hampshire Scholars' Repository. It has been accepted for inclusion in Space Science Center by an authorized administrator of University of New Hampshire Scholars' Repository. For more information, please contact Scholarly.Communication@unh.edu. 


\section{Authors}

H Bloemen, H deBoer, R Van Dijk, J W. den Herder, W Hermsen, L Kuiper, B Swanenburg, C de Vries, W Collmar, R Diehl, G G. Lichti, V Schonfelder, H Steinle, A W. Strong, Mark L. McConnell, A Connors, D Morris, G Stacy, and K Bennett 


\section{AIP $\mid$ Proceedings}

\section{COMPTEL observations of the inner galaxy}

H. Bloemen, H. de Boer, R. van Dijk, J. W. den Herder, W. Hermsen, L. Kuiper, B.

N. Swanenburg, C. P. de Vries, W. Collmar, R. Diehl, G. G. Lichti, V. Schönfelder,

H. Steinle, A. W. Strong, M. McConnell, A. Connors, D. Morris, G. Stacy, and K.

Bennett

Citation: AIP Conference Proceedings 280, 30 (1993); doi: 10.1063/1.44137

View online: http://dx.doi.org/10.1063/1.44137

View Table of Contents:

http://scitation.aip.org/content/aip/proceeding/aipcp/280?ver=pdfcov

Published by the AIP Publishing

\section{Articles you may be interested in}

Spectral modeling of gamma rays from black hole candidates

AIP Conf. Proc. 280, 418 (1993); 10.1063/1.44311

The number of positron sources in the galactic center region

AIP Conf. Proc. 280, 124 (1993); 10.1063/1.44158

Observations of galactic sources during Phase 1

AIP Conf. Proc. 280, 303 (1993); 10.1063/1.44136

Initial results from COMPTEL-an overview

AIP Conf. Proc. 280, 21 (1993); 10.1063/1.44123

The imaging and spectroscopy capabilities of the INTEGRAL imager: MonteCarlo simulation results

AIP Conf. Proc. 232, 489 (1991); 10.1063/1.40926 


\section{H. Bloemen ${ }^{1}$, H. de Boer, R. van Dijk ${ }^{2}$, J.W. den Herder, W. Hermsen, L. Kuiper, B.N. Swanenburg, C.P. de Vries \\ SRON-Leiden, P.O. Box 9504, 2300 RA Leiden, The Netherlands}

W. Collmar, R. Diehl, G.G. Lichti, V. Schönfelder, H. Steinle, A.W. Strong Max-Planck Institut für Extraterrestrische Physik, D-8046 Garching, F.R.G.

M. McConnell, A. Connors, D. Morris, G. Stacy ${ }^{3}$

Space Science Center, Univ. of New Hampshire, Durham, NH 03824, U.S.A.

K. Bennett

Astrophysics Division, ESTEC/ESA, 2200 AG Noordwijk, The Netherlands

1 Leiden Observatory, The Netherlands

2 Astronomical Institute, University of Amsterdam, The Netherlands

3 Compton Observatory Science Support Center, NASA GSFC, U.S.A.

\section{ABSTRACT}

This paper presents a first global study of COMPTEL observations of the inner Galaxy in the energy range $0.75-10 \mathrm{MeV}$. Preliminary findings demonstrate COMPTEL's capabilities for mapping the observed gamma radiation and disentangling the contributions from point sources and diffuse emission.

\section{INTRODUCTION}

The COMPTEL telescope aboard the Compton Observatory provides for the first time extensive imaging possibilities at $\mathrm{MeV}$ energies with a sourcelocation accuracy of typically $1^{\circ}$. This imaging capability is of particular value for studies of the Galactic disk, where a variety of point sources as well as diffuse emission can be expected to contribute. Strong et al. (this volume) found the total flux measured by COMPTEL from the central radian of the Galactic disk to be near the lower bound of a variety of previous flux estimates. This paper describes our initial attempts to map the observed emission and to disentangle the contributions from point sources and diffuse emission, using a combination of ten observations of the first and fourth Galactic quadrants obtained during the first half of the COMPTEL all-sky survey (Table 1). All analyses presented here were applied to three energy intervals, $0.75-1 \mathrm{MeV}, 1-3 \mathrm{MeV}$, and $3-10$ $\mathrm{MeV}$, but the findings for the latter two have been combined.

\section{MAPPING OF SOURCES AND DIFFUSE EMISSION}

Each incident photon detected by COMPTEL is first Compton scattered in the upper layer of detectors and then absorbed in the lower layer ${ }^{1}$. The energy deposits in the two layers define the Compton scatter angle (as well as the energy of the incident photon) and the interaction locations define the scatter direction. For a selected energy range, the photons can thus be binned in a 3-D dataspace 
Table 1. COMPTEL observations used in present analysis.

\begin{tabular}{lllll}
\hline Observation & \multicolumn{2}{l}{ Pointing } & \multicolumn{2}{l}{ Start } \\
& $\ell$ & $b$ & yy-mm-dd & $\begin{array}{l}\text { End } \\
\text { yy-mm-dd }\end{array}$ \\
\hline 2.0 & $73.3^{\circ}$ & $+2.6^{\circ}$ & $91-05-30$ & $91-06-08$ \\
5 & $0.0^{\circ}$ & $-4.0^{\circ}$ & $91-07-12$ & $91-07-26$ \\
7.0 & $70.4^{\circ}$ & $-8.3^{\circ}$ & $91-08-08$ & $91-08-15$ \\
7.5 & $25.0^{\circ}$ & $-14.0^{\circ}$ & $91-08-15$ & $91-08-22$ \\
12 & $310.7^{\circ}$ & $+22.2^{\circ}$ & $91-10-17$ & $91-10-31$ \\
13.0 & $25.0^{\circ}$ & $-14.0^{\circ}$ & $91-10-31$ & $91-11-07$ \\
$14^{*}$ & $285.0^{\circ}$ & $-0.7^{\circ}$ & $91-11-14$ & $91-11-28$ \\
16 & $0.0^{\circ}$ & $+20.2^{\circ}$ & $91-12-12$ & $91-12-27$ \\
$20^{*}$ & $39.7^{\circ}$ & $+0.8^{\circ}$ & $92-02-06$ & $92-02-20$ \\
$23^{*}$ & $322.1^{\circ}$ & $+3.0^{\circ}$ & $92-03-19$ & $92-04-02$ \\
\hline
\end{tabular}

* Only part of the available data included here.

consisting of the scatter direction and the scatter angle. The search for sources, model fitting, etc., are all carried out in this 3-D dataspace.

Two main imaging methods are applied: a maximum-entropy method for general imaging purposes ${ }^{2}$ and a likelihood method optimized to search for point sources $^{3}$ (including determination of statistical significance, flux, error region, etc.). This paper presents results from both methods.

The likelihood method in particular requires a careful estimate of the instrumental background, which is the dominant contributor to the total number of observed photons. The background models used in the present work are determined by applying a filter technique to the 3-D dataspace, which smooths the photon distribution and eliminates source signatures in first-order approximation. The likelihood algorithms for source mapping and model fitting use a modified instrument response to take this filtering into account. Formally, this technique is limited to point sources and line sources. It will be discussed in detail in a forthcoming paper.

\section{MODEL FITTING AND SIMULATIONS OF DIFFUSE EMISSION}

In order to illustrate the imaging potential of COMPTEL and to compare the observations with the results expected from diffuse emission, we also applied our mapping tools to Monte Carlo simulations of a simple model of the diffuse (bremsstrahlung) emission (see Strong et al. in this volume). This model is based on the assumption that the cosmic-ray (CR) electron density is constant throughout the Galaxy, so the $\gamma$-ray intensity distribution is approximately given by $I_{\gamma}=\varepsilon_{\gamma} N_{\mathrm{H}}$, where $N_{\mathrm{H}}$ is the column-density distribution of the interstellar gas (derived from various $\mathrm{HI}$ surveys and the $\mathrm{CO}$ survey by Dame et al. ${ }^{4}$, using a CO-to- $\mathrm{H}_{2}$ conversion factor of $2.3 \times 10^{20}$ molecules $\mathrm{cm}^{-2}\left(\mathrm{~K} \mathrm{~km} \mathrm{~s}^{-1}\right)^{-1}-$ see Fig. 1). First, the value of the $\gamma$-ray emissivity, $\varepsilon_{\gamma}$, was determined for each energy interval by maximum-likelihood fitting of the model to the binned photons in the $3-\mathrm{D}$ data space. The values are on average $\sim 20 \%$ lower than found by Strong et al., but considered to be in comfortable agreement in view of the different sets of observations used and the differing treatments of the background. Using these emissivities, simulated 3-D dataspaces of the diffuse emission were made, to which background simulations were added. 


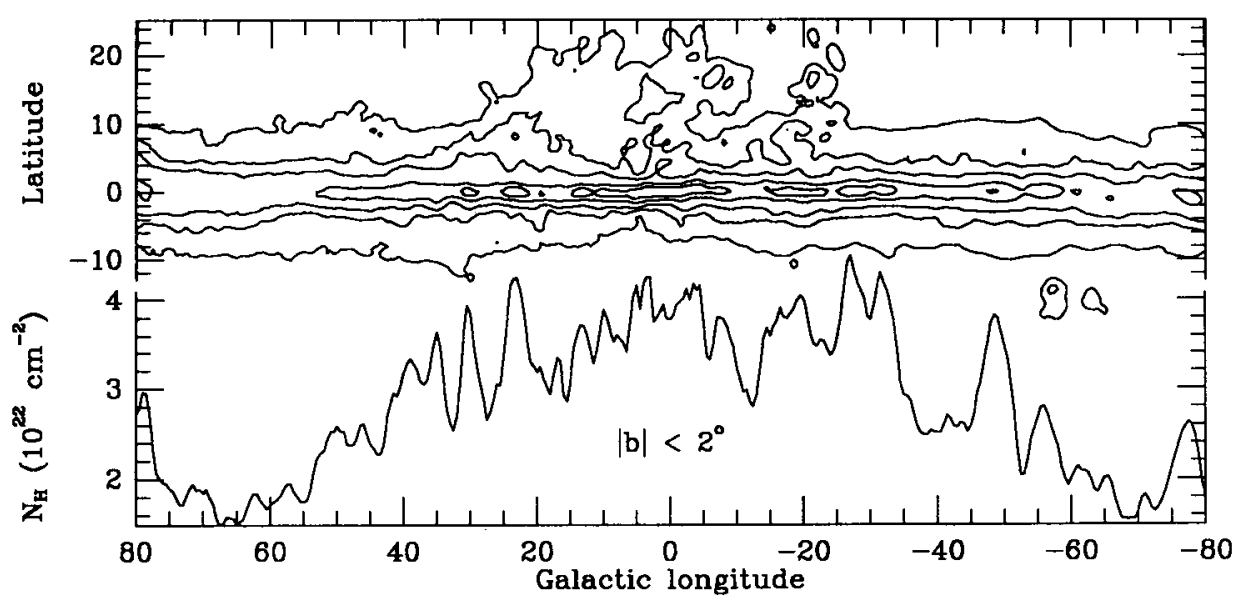

Figure 1: Part of the gas-column-density map (and longitude profile) used for model fitting and simulations of the diffuse $\gamma$-ray emission. Contour levels: 0.2 , $0.5,1,3$, and $6 \times 10^{22} \mathrm{~cm}^{-2}$. The $\mathrm{CO}$-to- $\mathrm{H}_{2}$ conversion factor for the strong $\mathrm{CO}$ emission from the Galactic centre was assumed to be ten times lower than for the Galactic disk ${ }^{5}$. The data were slightly smoothed for pictorial purposes.

\section{RESULTS}

Figures 2 and 3 show our mapping results in the energy ranges $1-10 \mathrm{MeV}$ and $0.75-1 \mathrm{MeV}$, both for the actual observations (top) and for the simulations of the diffuse emission (bottom). Only likelihood maps are shown for the $0.75-1$ $\mathrm{MeV}$ range - the maximum-entropy maps are very similar.

The likelihood maps show the quantity $-2 \ln \lambda$, where $\lambda$ is the maximum likelihood ratio $L$ (background) / $L$ (source+background). Formally, in a search for sources, $-2 \ln \lambda$ has a chi-square distribution with 3 degrees of freedom (for instance, a $3 \sigma$ detection corresponds to $-2 \ln \lambda=13.8$ ). Simulations have shown, however, that our method of background modelling requires a somewhat more stringent criterion for source detection (discussed in a future paper).

Figures 2 and 3 give quite a different impression of the global appearance of the gamma-ray sky in the two energy bands. Fig. 2 (1-10 MeV) shows a clear ridge of emission. The likelihood maps and maximum-entropy maps are very similar, the latter showing more detailed structure because of the deconvolution inherent in the maximum-entropy method. The global appearance of the observations is similar to that of the simulated observations of the diffuse emission, but detailed differences seem to be present.

In Fig. $3(0.75-1 \mathrm{MeV})$, only a few excesses are seen in addition to those at the positions of the black-hole candidate Cyg X-1 and the radio galaxy Cen A (both at flux levels at least an order of magnitude below some previous detections - see McConnell et al. and Collmar et al. in this volume). Although one of the other excesses coincides with the Galactic-centre source 1E1740.7-2942 (with a flux of $\sim 1 \times 10^{-4} \mathrm{~cm}^{-2} \mathrm{~s}^{-1}$, at least an order of magnitude above extrapolations of all published spectra of this source), it cannot be excluded that this excess is actually due to a peak in the diffuse emission, visible in the lower map of Fig. 3. Only a few features due to diffuse emission can apparently be expected to be seen by COMPTEL in this narrow energy band. The observed excesses 

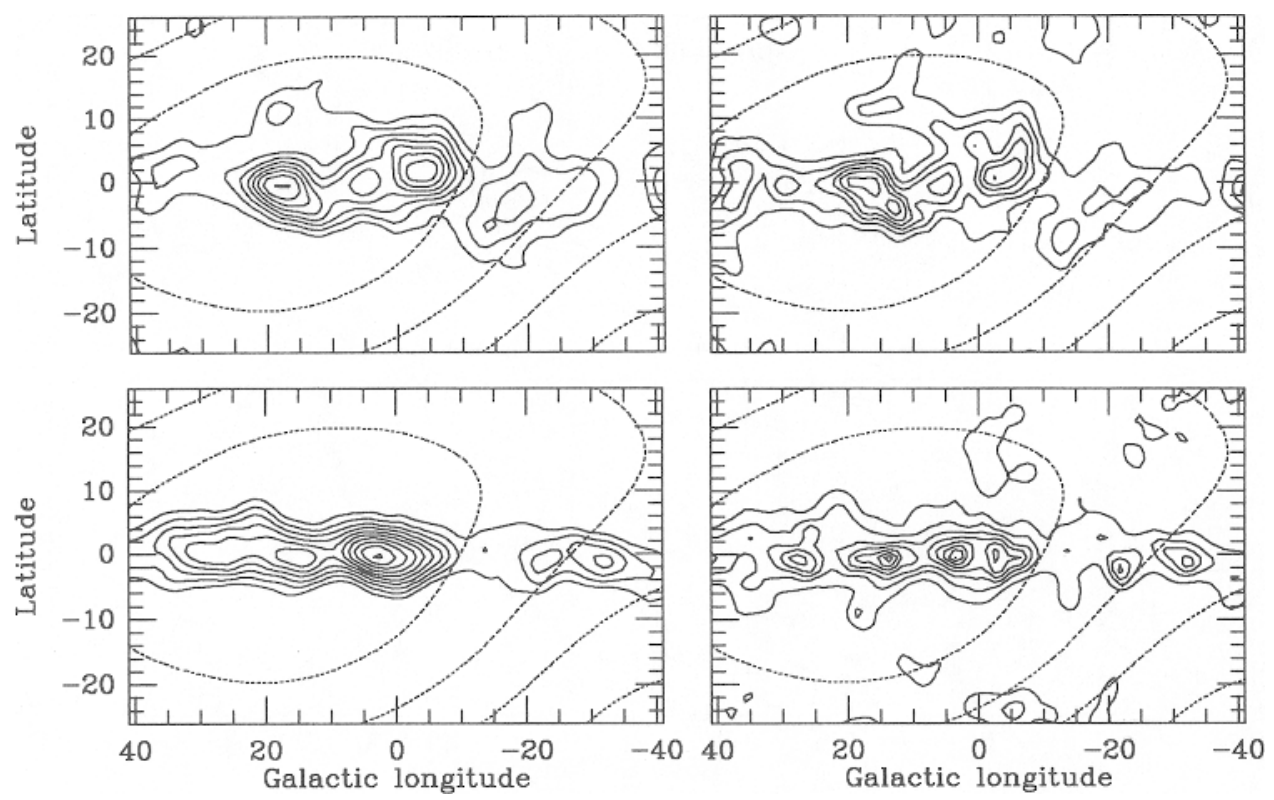

Figure 2: Top: Likelihood map (left) and maximum-entropy map (right) of combined COMPTEL observations (Table 1) of the inner Galaxy (1-10 MeV). Bottom: Same for simulated observations of the diffuse emission, with emissivity from model fitting. Contour levels: (left) start 15, step 12 ; (right) start $4 \times 10^{-3}$, step $3 \times 10^{-3} \mathrm{~cm}^{-2} \mathrm{~s}^{-1} \mathrm{sr}^{-1}$. Contours of effective exposure, at $20 \%$ intervals from the peak value, are superimposed.

only partly coincide with the expected diffuse excesses. This may either hint at the presence of sources (or local CR-density enhancements), implying that we overestimated the contribution from diffuse emission in the model fitting, or it may be attributable to the limitations of our simple diffuse model.

The obvious question is whether the apparent differences between observations and simulations are indeed significant. This was studied by including the diffuse model in the likelihood search for sources. Fig. 4 shows the resulting map of $-2 \ln \lambda$, where $\lambda$ is now the maximum likelihood ratio $L$ (diffuse+background) / $L$ (source+diffuse+background). Two main excesses can be seen. The one near $\ell=15^{\circ}-20^{\circ}$ coincides with a similar extended feature seen in both the COS-B data $^{6}$ and the EGRET data (Mayer-Hasselwander et al., this volume). There is no obvious counterpart for the second excess $\left(\ell \simeq 345^{\circ}, b \simeq-6^{\circ}, 95 \%\right.$ error radius of $\sim 1.5^{\circ}$ ); its position is consistent with that of the LMXRB 1735-44. The location of these 'sources' outside the Galactic disk may indicate that an ensemble of sources along the plane is hidden because we have overestimated the contribution from diffuse emission. This issue will be addressed in future work by fitting a number of sources simultaneously with diffuse emission.

It can be concluded that COMPTEL's potential to disentangle the $\mathrm{MeV}$ emission from the inner Galaxy is promising. We emphasize, however, that this paper should mainly be regarded as a summary of our analysis strategy; the results are preliminary. 


\section{COMPTEL Observations of the Inner Galaxy}
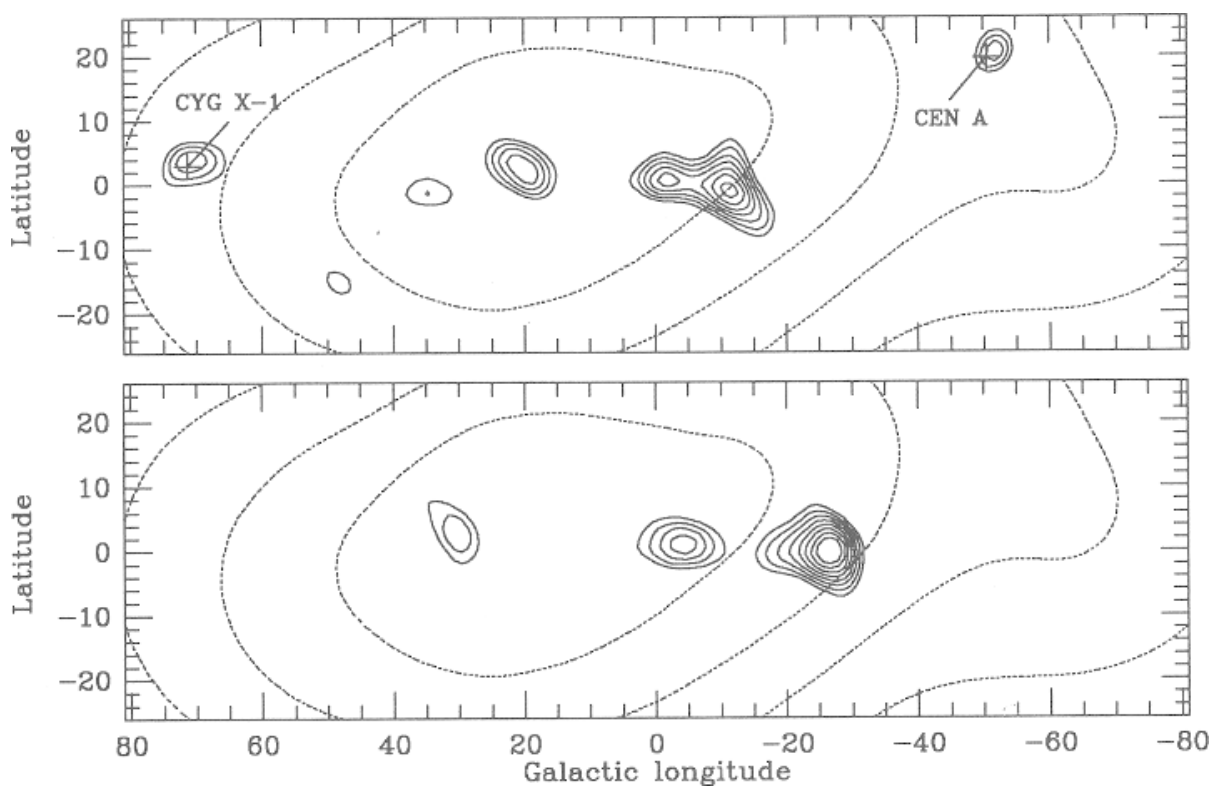

Figure 3: Top: Likelihood map (0.75-1 MeV) of combined COMPTEL observations of the inner Galaxy (Table 1). Bottom: Same for simulated observations of the diffuse emission, with emissivity from model fitting. Contour levels: start at $-2 \ln \lambda=15$, step 5. Exposure contours as in Fig. 2.

Figure 4:

Likelihood map $(0.75-10 \mathrm{MeV})$ showing enhanced emission not explained by our model of the diffuse emission (see text). This map is a sum of the maps for the three individual energy intervals. Contours: start 20, step 8.

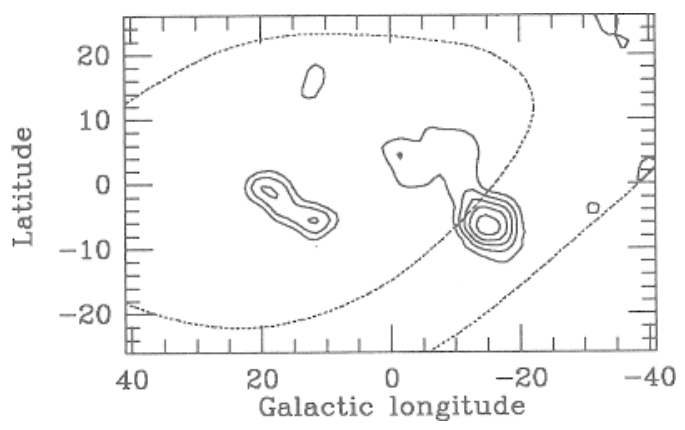

\section{REFERENCES}

1. V. Schönfelder et al., Ap. J. Suppl., in press (1993)

2. A.W. Strong et al., in Data Analysis in Astronomy IV, eds. V. Di Gesù et al., Plenum Press, New York (1992)

3. H. de Boer et al., in Data Analysis in Astronomy IV (see ref. 2), p. 241

4. T.M. Dame et al., Ap. J. 322, 706 (1987)

5. L. Blitz et al., A\&A 143, 267 (1985)

6. H. Bloemen, in The Evolution of the Interstellar Medium, ed. L. Blitz, ASP Conference Series, p. 79 (1990) 